Chirurg 2014 · 85:349

DOI 10.1007/s00104-014-2727-9

Online publiziert: 14. März 2014

C Springer-Verlag Berlin Heidelberg 2014
O. Strobel · M.W. Büchler

Klinik für Allgemein-, Viszeral- und Transplantationschirurgie, Universität Heidelberg

\section{Parenchymdurchtrennung mittels Stapler vs. Clamp-Crushing bei Leberresektion}

\section{Eine randomisierte kontrollierte Studie}

\section{Originalpublikation}

Rahbari NN, Elbers H, Koch M et al (2014) Randomized clinical trial of stapler versus clamp-crushing transection in elective liver resection. Br J Surg 101:200-207

\section{Hintergrund und Fragestellung}

Die Parenchymdurchtrennung ist bei der Leberresektion ein zentraler Schritt, der Auswirkungen auf den intraoperativen Blutverlust und auf die Rate der wichtigsten postoperativen Komplikationen hat. Neu entwickelte Techniken und Geräte zur Parenchymdurchtrennung zeigten bisher im Vergleich zu der von den meisten Autoren als Standard akzeptierten klassischen Clamp-CrushingTechnik keine Überlegenheit. Die Parenchymdurchtrennung mittels vaskulären Staplern wurde in retrospektiven Analysen als effektives und sicheres Verfahren beschrieben, das durch eine kurze Phase der Parenchymdurchtrennung zur Reduktion des Blutverlustes führen kann. Rahbari et al. untersuchten nun in einer randomisierten kontrollierten Studie die Parenchymdurchtrennung mittels Stapler vs. Clamp-Crushing bei elektiven Leberresektionen.

\section{Methoden}

Zwischen 01/2010 und 10/2011 wurden 442 Patienten mit geplanter Leberresektion gescreent und 130 Patienten intraoperativ in die Gruppen Clamp-Crushing $(n=65)$ und Stapler $(\mathrm{n}=65)$ randomisiert. $312 \mathrm{~Pa}$ tienten wurden ausgeschlossen, davon 89 (20,1\%) weil eine Parenchymdurchtrennung mittels Stapler technisch nicht möglich war. Primärer Endpunkt der Studie war der intraoperative Blutverlust. Als sekundäre Endpunkte wurden Dauer der Parenchymdurchtrennung und der gesamten Operation, Komplikationsraten und Resektionsränder untersucht.

\section{Ergebnisse}

Die beiden Gruppen waren bezüglich epidemiologischer Parameter, Diagnose, Beschaffenheit des Parenchyms, präoperativer Laborparameter sowie bezüglich der Art und der Ausmaße der durchgeführten Resektionen vergleichbar. Während der gesamte intraoperative Blutverlust bei Clamp-Crushing und Stapler vergleichbar war (1050 vs. $925 \mathrm{ml}$ ), war der Blutverlust während der Phase der Parenchymdurchtrennung in der Stapler-Gruppe signifikant niedriger (400 vs. $200 \mathrm{ml}$ ). Sowohl die Dauer der gesamten Operation (221 vs. $190 \mathrm{~min}$ ) als auch die Dauer der Parenchymdurchtrennung ( 30 vs. 9 min) waren in der Stapler-Gruppe signifikant kürzer. Sowohl bezüglich der Rate und des Schweregrad der Komplikationen als auch speziell hinsichtlich der wichtigsten Komplikationen Galleleck und Blutung gab es keine Unterschiede zwischen den Gruppen. Auch die postoperative Krankenhausverweildauer (10 vs. 10 Tage) und die Rate an positiven Absetzungsrändern (7 vs. $8 \%$ ) waren vergleichbar.

\section{Diskussion}

Die Studie zeigt, dass die Parenchymdurchtrennung mittels Stapler sicher und effektiv ist. Im Vergleich zum ClampCrushing führt die Stapler-Technik zu einer Verkürzung der Phase der Parenchymdurchtrennung und zu einer Verminderung des Blutverlustes in dieser Phase sowie zu einer Verkürzung der Operationszeit.

Anhand einer Subgruppenanalyse vermuten die Autoren, dass diese Vorteile der Stapler-Technik vor allem bei anatomischen Majorresektionen zum Tragen kommen. Ein weiterer Vorteil der Stapler-Technik könnte in einer verminderten Notwendigkeit und verkürzten Dauer einer vaskulären Ausklemmung insbesondere bei vorgeschädigten Lebern liegen. Diese Aspekte müssen in weiterführenden Studien untersucht werden.

\section{Korrespondenzadresse}

PD Dr. 0. Strobel

Klinik für Allgemein-, Viszeralund Transplantationschirurgie, Universität Heidelberg, Im Neuenheimer Feld 110, 69120 Heidelberg Oliver.Strobel@med.uni-heidelberg.de

Interessenkonflikt. O. Strobel und M.W. Büchler geben an, dass kein Interessenkonflikt besteht. 